\title{
Pneumococcal vaccination and otitis media in Australian Aboriginal infants: comparison of two birth cohorts before and after introduction of vaccination
}

\author{
Grant Austin Mackenzie*1,2,3,4, Jonathan Rhys Carapetis ${ }^{2,3}$, \\ Amanda Jane Leach ${ }^{2,3}$ and Peter Stanley Morris $2,3,5$
}

Address: ${ }^{1}$ Bacterial Diseases Program, Medical Research Council (UK) Laboratories, Fajara, Gambia, ${ }^{2}$ Child Health Division, Menzies School of Health Research, Darwin, NT, Australia, ${ }^{3}$ Institute for Advanced Studies, Charles Darwin University, Darwin, NT, Australia, ${ }^{4}$ School of Medicine, Flinders University of South Australia, Adelaide, SA, Australia and ${ }^{5}$ NT Clinical School, Flinders University of South Australia, Darwin, NT, Australia

Email: Grant Austin Mackenzie* - gmackenzie@mrc.gm; Jonathan Rhys Carapetis - jonathan.carapetis@menzies.edu.au; Amanda Jane Leach - amanda.leach@menzies.edu.au; Peter Stanley Morris - peter.morris@menzies.edu.au

* Corresponding author

Published: 19 February 2009

BMC Pediatrics 2009, 9:14 doi:10.1/86/147|-2431-9-14

This article is available from: http://www.biomedcentral.com/|47|-243I/9//4

(c) 2009 Mackenzie et al; licensee BioMed Central Ltd.

This is an Open Access article distributed under the terms of the Creative Commons Attribution License (http://creativecommons.org/licenses/by/2.0), which permits unrestricted use, distribution, and reproduction in any medium, provided the original work is properly cited.
Received: 26 October 2008

Accepted: 19 February 2009

\begin{abstract}
Background: Aboriginal children in remote Australia have high rates of complicated middle ear disease associated with Streptococcus pneumoniae and other pathogens. We assessed the effectiveness of pneumococcal vaccination for prevention of otitis media in this setting.

Methods: We compared two birth cohorts, one enrolled before (I996-200I), and the second enrolled after introduction of 7-valent pneumococcal conjugate and booster 23-valent polysaccharide vaccine (200I-2004). Source populations were the same for both cohorts. Detailed examinations including tympanometry, video-recorded pneumatic otoscopy and collection of discharge from tympanic membrane perforations, were performed as soon as possible after birth and then at regular intervals until 24 months of life. Analyses (survival, point prevalence and incidence) were adjusted for confounding factors and repeated measures with sensitivity analyses of differential follow-up.

Results: Ninety-seven vaccinees and 5 I comparison participants were enrolled. By age 6 months, 96\% $(8 I / 84)$ of vaccinees and $100 \%(4 I / 4 I)$ of comparison subjects experienced otitis media with effusion (OME), and by 12 months $89 \%$ and $88 \%$ experienced acute otitis media (AOM), $34 \%$ and $35 \%$ experienced tympanic membrane perforation (TMP) and I4\% and $23 \%$ experienced chronic suppurative otitis media (CSOM). Age at the first episode of OME, AOM, TMP and CSOM was not significantly different between the two groups. Adjusted incidence of AOM (incidence rate ratio: 0.88 [95\% confidence interval $(\mathrm{Cl})$ : 0.69-I.I3]) and TMP (incidence rate ratio: 0.63 [0.36-I.II]) was not significantly reduced in vaccinees. Vaccinees experienced less recurrent TMP, 9\% (8/95) versus 22\% (I I/5 I), (odds ratio: 0.33 [0. II-I.00]).

Conclusion: Results of this study should be interpreted with caution due to potential bias and confounding. It appears that introduction of pneumococcal vaccination among Aboriginal infants was not associated with significant changes in prevalence or age of onset of different OM outcomes or the incidence of AOM or TMP. Vaccinees appeared to experience reduced recurrence of TMP. Ongoing high rates of complicated OM necessitate additional strategies to prevent ear disease in this population.
\end{abstract}




\section{Background}

Young Aboriginal children in remote Australia have a $24 \%$ prevalence of tympanic membrane perforation (TMP) and 15\%[1] to 24\%[2] prevalence of chronic suppurative otitis media (CSOM). The World Health Organization states that CSOM prevalence greater than $4 \%$ indicates a massive public health problem[3].

Among Aboriginal infants, Haemophilus influenzae, Streptococcus pneumoniae, and Moraxella catarrhalis have been isolated from $57 \%, 34 \%$ and $4 \%$ of TMPs respectively, with H. influenzae and S. pneumoniae co-infection in $28 \%$ of cases[4]. Pneumococcal serotypes associated with TMP are 19A, 19F, 23F, 14 and 1[5]. The 7-valent pneumococcal conjugate vaccine (7PCV) includes three of these: $19 \mathrm{~F}$, $23 \mathrm{~F}$ and 14 as well as serotypes $4,6 \mathrm{~B}, 9 \mathrm{~V}$ and $18 \mathrm{C}$. Trials of 7PCV suggest efficacy of $6 \%$ against acute otitis media (AOM) in American and Finnish children[6,7] with greater efficacy against more severe outcomes (9\%[7] to $10 \%[6]$ against recurrent AOM and 23\%[8] to 39\%[9] against tympanostomy procedures). Two studies have reported $100 \%$ efficacy against TMP due to vaccine serotypes, excluding $19 \mathrm{~F}[8,10]$. Efficacy against any TMP or CSOM is unknown. We thought that 7PCV might be even more effective in populations with a high burden of severe otitis (Australian Aboriginal children and others with substantial prevalence of CSOM [11-14]). Pneumococcal vaccination for Aboriginal children in Australia began in late 2001. We compared: 1) time to develop otitis media with effusion (OME) and other OM outcomes, 2) OM prevalence outcomes and 3) OM incidence outcomes in two birth cohorts of Aboriginal infants, before and after introduction of pneumococcal vaccination.

\section{Methods \\ Study Setting}

Two cohorts were enrolled from three Aboriginal communities on the Tiwi islands north of Darwin (population $2,029)[15]$. We compared a cohort that received pneumococcal vaccination (2001-2004) with a comparison cohort from the same communities prior to vaccine availability (1996-2001).

\section{Participants}

The comparison, or before vaccine group, was enrolled in a randomised controlled trial of long-term amoxicillin versus placebo between 1996 and 2001 (OM-RCT)[16]. As subjects enrolled in the OM-RCT began randomised therapy after detection of the first OME episode, the comparison group for the time to first OME outcome included all subjects enrolled in the OM-RCT. For all other outcomes, the comparison group comprised only those participants assigned to placebo. The vaccinated group was enrolled between 2001 and 2004, after catch-up vaccina- tion for those less than 2 years of age, and introduction of routine pneumococcal vaccination in July 2001.

Comparison group exclusion criteria were: age $>12$ months, gestation $<34$ weeks, penicillin sensitivity, longterm antibiotic therapy, craniofacial abnormality, CSOM and immunodeficiency[16]. Exclusion criteria for the vaccinated group were: age $>4$ months, gestation $<34$ weeks and congenital abnormality.

\section{Consent and Ethical Considerations}

The mother or carer gave written informed consent for each study. The Institutional Ethics Committee of Territory Health Services and Menzies School of Health Research, and the Tiwi Health Board approved the studies.

\section{Procedures}

Comparison participants were enrolled as soon as possible after birth and examined every two weeks until OME was detected. They were then randomised to amoxicillin or placebo until middle ear status was normal. Children received randomised therapy and monthly examinations for an average of 5.2 months[16]. Vaccinees were also enrolled as soon as possible after birth and examined every two weeks until OME was detected, and then monthly until 12 months of age. Both groups were examined at 12, 18 and 24 months of age or until study completion.

All participants received standard care, including vaccinations, from community clinics. Vaccinees were scheduled to receive 7PCV at 2, 4 and 6 months of age and 23-valent polysaccharide vaccine (23PPV) at 18 months. The research team provided additional clinical care if new problems were identified during assessment. Medications provided by the study and community clinic were supervised for the comparison group but not for vaccinees. Standard antibiotic therapy for AOM among the vaccinated cohort was amoxicillin $50 \mathrm{mg} / \mathrm{kg} /$ day for 7 days (research team) and $300 \mathrm{mg}$ twice daily for 5 days (community clinic), and for the comparison group, amoxicillin $50 \mathrm{mg} / \mathrm{kg} /$ day for 5 days (research team) and $125 \mathrm{mg}$ thrice daily for 5 days (community clinic).

Assessment involved direct and video-recorded pneumatic otoscopy, tympanometry (GSI 38 Auto-Tymp Grayson-Stadler) and review of clinic records. Two independent, unblinded assessors standardised video diagnoses in the two cohorts. Consensus between assessors' video diagnoses was required in both studies and if a diagnosis in the 1996-2001 cohort was changed. If assessors disagreed a third assessor resolved the decision. Data were recorded using standard forms. 


\section{Definitions}

- OME: Type B tympanogram with neutral or mild bulging of the TM.

- AOM: Moderate or marked bulging of the TM. New episodes were defined when the preceding examination was OME or normal.

- TMP: Discharge through a perforation for $<6$ weeks or pus in the auditory canal. New episodes were defined when the preceding examination was AOM, OME or normal.

- CSOM: TMP with discharge for 6 weeks or greater.

\section{Microbiology of Tympanic Membrane Perforations} Microbiological techniques were the same for both groups. Aluminium-shafted, cotton-tipped swabs (Disposable Products, Australia) of perforation discharge were collected through, or from as close as possible to, the site of perforation. Specimens were transported and stored frozen as recommended for pneumococcal carriage studies[17]. Aliquots $(10 \mu \mathrm{l})$ were cultured on selective media and incubated overnight at $37^{\circ} \mathrm{C}$ in $5 \% \mathrm{CO}_{2}$. S. pneumoniae was confirmed with serotyping by the Quellung reaction, $H$. influenzae by dependence on $\mathrm{X}$ and $\mathrm{V}$ factors, and M. catarrhalis by colony morphology, Gram stain and oxidase production.

\section{Statistical Analysis}

Vaccinee and placebo recipient data were compared, apart from the time to first OME outcome, for which comparison group data were included from all randomised subjects in the OM-RCT; i.e. before detection of OME and subsequent randomisation.

For time to first OME and AOM, data were included if the first examination was before 4 months of age. For time to first TMP and CSOM, data were included if the first examination was before 6 and 9 months of age respectively. Participant data were censored if the duration between consecutive examinations was greater than 2 months. Examinations after 24 months of age were excluded. To explore potential effects of unobserved clinical events because of differential follow-up, sensitivity analyses were performed including different ages at first examination (range less than 4 months to any age) and examination intervals (less than 2 to 6 months).

Analyses of pathogens associated with new perforation (specimen collected $<28$ days following new perforation) were performed per ear rather than per child. Recurrent ( $>1$ episode in one ear) or bilateral perforation during follow-up were examined by log-linear modelling.
Time to first event outcomes used Kaplan-Meier curves and Cox proportional hazards models. Incidence outcomes used Poisson regression with estimates adjusted for repeated measures using generalised estimating equations. A priori analysis included adjustment for 'rate of non-respiratory illness' (incidence of presentations for diarrhoea, anaemia, failure to thrive, skin sores, scabies and fungal infection) as a measure of general ill health between studies. Other potentially confounding variables (gender, age at first examination, rate of well and sick clinic visits, rate of prior antibiotic prescription, birth weight, delayed immunisation ( $>1$ month after due date) were included in forward stepwise modeling if p-values were less than 0.10. Statistical significance was the $5 \%$ confidence level. Assuming a baseline median time to OME of 60 days[18], an alpha level of 0.05 with 100 vaccinated and 80 comparison participants, the comparison had power of 0.77 to detect a clinically important difference of 20 days. Assuming a baseline cumulative proportion experiencing TMP of 50\%, alpha of 0.05 with 100 vaccinated and 50 comparison participants, the comparison had power of 0.82 to detect an expected reduction of $50 \%$ to a cumulative proportion of $25 \%$.

\section{Role of the Funding Source}

The National Health \& Medical Research Council of Australia funded the RCT. Wyeth Australia funded the vaccine study. Neither was involved in study design, collection, analysis, interpretation of data, writing or submission of the report.

\section{Results \\ Enrolment and Comparability of Groups}

Greater than $80 \%$ of infants in the population after vaccine introduction were enrolled and 54\%[16] were enrolled in the before vaccination cohort. There were 97 vaccinees (mean follow-up, 470 days [range 35-728]; Figure 1) and 51 comparison children randomised to placebo (mean follow-up, 472 days [range 154-727]). Mean number of examinations to age 24 months was 10.9 (range 1-22) for vaccinees and 9.5 (range 3-17) for comparison participants. Vaccinees began follow-up at a younger age than comparison participants but by eight months of age similar proportions of each group had begun follow-up (Figure 1). Among vaccinees the third dose of $7 \mathrm{PCV}$ was given at a mean age of 6.7 months (range 4.2, 13.6) and at greater than 8 months for $14 / 93$ (15\%). Sex distribution, birth weight and maternal age were similar among the comparison groups (Table 1). Compared to comparison participants in the first 6 months of life, vaccinees had reduced rates of lower respiratory illness, were younger at first examination, had fewer well clinic visits, and fewer were late for immunisation (Table 2). Although the reduced rate of lower respira- 
Table I: Vaccinated and comparison group characteristics

\begin{tabular}{llll}
\hline Participant Characteristic & $\begin{array}{l}\text { Vaccinated } \\
\mathbf{N = 9 7}\end{array}$ & $\begin{array}{l}\text { Comparison } \\
\mathbf{N}=\mathbf{5} \mathbf{I}\end{array}$ & Mean Difference or Odds Ratio (95\% Cl) \\
\hline Sex (\% Male) & $45(46 \%)$ & $30(59 \%)$ & $0.6 \mathrm{I}(0.29,1.27)$ \\
Birth weight (g) & $2956(\mathrm{n}=87)$ & $3159(\mathrm{n}=49)$ & $-203(-409,3)$ \\
Maternal age (years) & $24.2(\mathrm{n}=95)$ & $23.0(\mathrm{n}=49)$ & $1.2(-0.8,3.1)$ \\
\hline
\end{tabular}

Birth weight was missing for 10 vaccinees and 2 comparison participants. Maternal age was missing for 2 vaccinees and 2 comparison participants.

tory illness among vaccinees may be attributable to pneumococcal vaccination, and not potential confounding factors, the other characteristics in Table 2 may still be used for comparison of the two groups.

Comparing census data from 2001 and 1996, there were similar income levels, proportions of residents aged 0-4, and rates of unemployment (data not shown). Average household occupancy was lower in 2001 than 1996, 4.5 versus 5.1 occupants per house. $[15,19]$

\section{Prevalence of Otitis Media Outcomes in the First Year of Life}

By 2 months of age approximately $70 \%$ of both vaccinated and comparison participants had developed OME, by 6 months virtually all had developed OME (Table 3). By 12 months, approximately 90\% in both groups had documented AOM. The proportion of vaccinees experiencing TMP in the first 9 months of life was lower than comparison participants, although by 12 months $35 \%$ of both groups had experienced TMP. By age 12 months, $14 \%$ of vaccinees and $23 \%$ of comparison participants had developed CSOM (Table 3). Seven children excluded from the OM-RCT due to a diagnosis of CSOM at initial assessment[16] were not included in this study, although their exclusion may have reduced the proportion of comparison participants at risk of developing CSOM.

\section{Time to First Otitis Media Outcomes}

Time to first OME analysis included 90 vaccinees and 70 comparison participants. Univariate and multivariate analyses showed time to first OME was not significantly different by group (Table 4). Increased time to first OME was associated with older age at first examination. Univariate and multivariate analyses indicated that vaccination had no effect on time to first AOM, TMP or CSOM (Table 4). Kaplan-Meier curves were similar for both groups (Figure 2).

\section{Unadjusted, Multivariate, and Sensitivity Analyses of the Incidence of Acute Otitis Media and Tympanic Membrane Perforation}

Analysis of AOM incidence included 90 vaccinees and 37 comparison participants. Unadjusted analysis showed vaccination associated with an AOM absolute rate reduction (ARR) of 0.18 episodes per person-year ( 2.05 in com-

Table 2: Clinical characteristics of vaccinated and comparison groups in the first 6 months of life

\begin{tabular}{llllc}
\hline Clinical Characteristic & $\begin{array}{l}\text { Vaccinated } \\
\mathbf{N = 9 4}\end{array}$ & $\begin{array}{l}\text { Comparison } \\
\mathbf{N = 4 1}\end{array}$ & Mean Difference or Odds Ratio (95\% CI) \\
\hline \multirow{2}{*}{ Mean } & Age at first examination (in days) & 41 & 60 & $-18(-31--5)$ \\
& Well clinic visits & 4.84 & 10.3 & $-5.48(-7.21--3.75)$ \\
& Sick clinic visits & 6.95 & 8.90 & $-1.96(-4.46-0.55)$ \\
& Upper respiratory illnesses* & 1.33 & 1.00 & $0.33(-0.21-0.87)$ \\
& Lower respiratory illnesses & 0.98 & 3.21 & $-2.24(-2.89--1.59)$ \\
& Non-respiratory illnesses & 2.80 & 2.71 & $0.09(-1.06-1.24)$ \\
& Diarrhoeal illnesses & 0.62 & 0.85 & $-0.24(-0.76-0.28)$ \\
& Antibiotic prescriptions & 3.54 & 4.51 & $-0.97(-2.30-0.37)$ \\
\hline \multirow{2}{*}{ Proportion } & & $5(5 \%)$ & $1(2 \%)$ & $2.25(0.24-109)$ \\
& Failure to thrive & $27(29 \%)$ & $12(29 \%)$ & $1.02(0.45-2.31)$ \\
& External nasal discharge & $18(19 \%)$ & $9(22 \%)$ & $0.84(0.32-2.37)$ \\
& Admitted to hospital & $14(15 \%)$ & $18(44 \%)$ & $0.22(0.09-0.56)$ \\
& Late immunisation $\dagger$ & $24 \%$ & $36 \%$ & $-12(-40-15)$
\end{tabular}

Note: 3 vaccinated and 10 comparison participants were excluded from clinical comparison in the first 6 months of life as their first examinations occurred at greater than 6 months of age.

*Upper respiratory illness includes OM or other upper respiratory tract infections.

tImmunisation greater than one month after due date.

$\ddagger$ Anaemia defined as haemoglobin level less than $9 \mathrm{~g} / \mathrm{dL}$. 

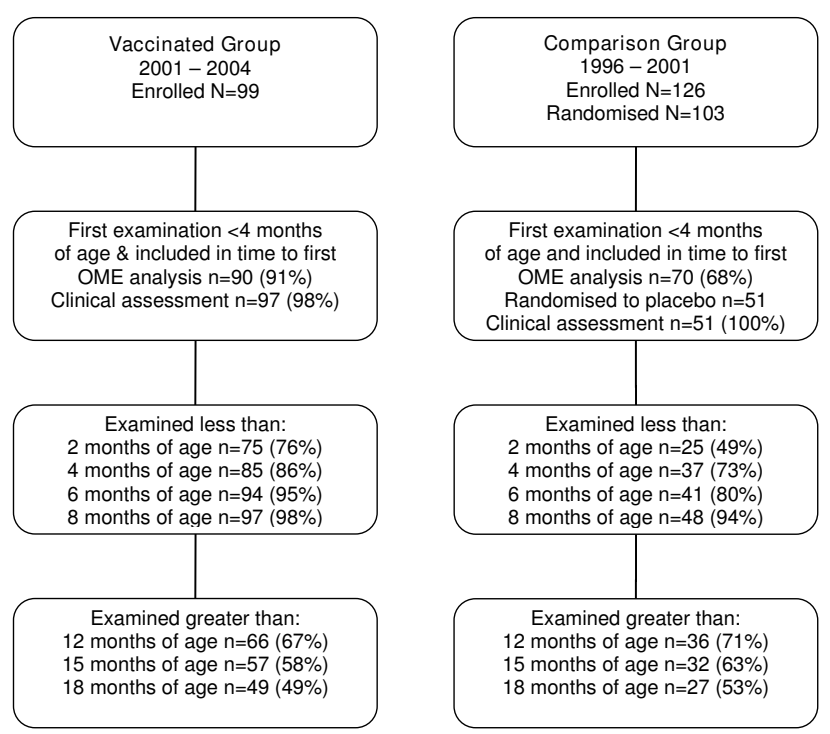

Figure I

Enrolment and follow-up profile. Demonstrating numbers of participants entering and leaving follow-up at different ages.

parison and 1.87 in vaccinated participants; incidence rate ratio (IRR): 0.91 [95\% CI: 0.64-1.29]). Increased AOM incidence was associated with the $a$ priori adjustment variable, non-respiratory illness rate, which was included in multivariate modelling (data not shown). Vaccination was associated with an adjusted ARR of 0.20 episodes per person-year (2.03 in comparison and 1.83 in vaccinated participants; IRR: 0.88 [95\% CI: 0.69-1.13]). Sensitivity analysis including any age at first examination and less than 4 month examination intervals (97 vaccinees, 51 comparison participants) resulted in a different adjusted ARR of 0.40 episodes per person-year (2.02 in comparison and 1.62 in vaccinated participants; IRR: 0.81 [95\% CI 0.67-0.99]). Recurrent AOM (>1 episode) occurred in $45 \%(44 / 97)$ of vaccinees and 55\% (28/51) of comparison participants (Table 5).

Analysis of the incidence of new perforation included 94 vaccinees and 41 comparison participants. Unadjusted analysis showed vaccination associated with an ARR of new perforation of 0.27 episodes per person-year $(0.82$ in comparison and 0.55 in vaccinated participants; IRR: 0.68 [95\% CI 0.40-1.14]). Increased incidence of new perforation was associated with increased antibiotic prescription and non-respiratory illness rate (data not shown). Multivariate modelling showed vaccination associated with an adjusted ARR of 0.28 episodes per person-year ( 0.75 in comparison and 0.47 in vaccinated participants; IRR: 0.65 [95\% CI: 0.38-1.10]). Sensitivity analysis including any age at first examination and less than 4 month examina-
Table 3: Cumulative proportions of participants over time with different otitis outcomes

\begin{tabular}{|c|c|c|c|}
\hline Outcome & $\begin{array}{c}\text { Age } \\
\text { (months) }\end{array}$ & $\begin{array}{c}\text { Vaccinated } \\
\text { n/N (\%) }\end{array}$ & $\begin{array}{c}\text { Comparison } \\
n / N(\%)\end{array}$ \\
\hline \multirow[t]{4}{*}{ OME } & I & $27 / 47$ (57) & $6 / 13(46)$ \\
\hline & 2 & $49 / 73(67)$ & $18 / 25(72)$ \\
\hline & 4 & $72 / 83$ (87) & $35 / 37$ (95) \\
\hline & 6 & $81 / 84(96)$ & $4 I / 4 I(100)$ \\
\hline \multirow[t]{5}{*}{ AOM } & 2 & $5 / 73(7)$ & $4 / 25(16)$ \\
\hline & 4 & $33 / 83(40)$ & $19 / 37(5 \mathrm{I})$ \\
\hline & 6 & $53 / 84(63)$ & $33 / 4 I(80)$ \\
\hline & 9 & 65/83 (78) & $4 I / 45$ (9I) \\
\hline & 12 & 65/73 (89) & $35 / 40(88)$ \\
\hline \multirow[t]{6}{*}{ TMP } & 4 & $3 / 83(4)$ & 2/37 (5) \\
\hline & 6 & $10 / 84(12)$ & $8 / 41$ (20) \\
\hline & 9 & I8/83 (22) & $14 / 45(31)$ \\
\hline & 12 & $25 / 73(34)$ & $14 / 40(35)$ \\
\hline & 15 & $20 / 59$ (38) & 14/35 (40) \\
\hline & 24 & $15 / 30(50)$ & $12 / 23(52)$ \\
\hline \multirow[t]{5}{*}{ CSOM } & 6 & $\mathrm{I} / 84(\mathrm{I})$ & $3 / 4 \mid(7)$ \\
\hline & *9 & 4/83 (5) & $8 / 45$ (18) \\
\hline & 12 & $10 / 73(14)$ & $9 / 40(23)$ \\
\hline & 15 & $8 / 59(14)$ & $7 / 35(20)$ \\
\hline & 24 & $9 / 30(30)$ & $9 / 23$ (39) \\
\hline
\end{tabular}

To minimise loss of case ascertainment the denominator includes only participants followed to the age of the given category. $*_{p}<0.05$

tion intervals (97 vaccinees, 51 comparison participants) resulted in a different adjusted ARR of 0.36 episodes per person-year ( 0.75 in comparison and 0.39 in vaccinated participants; IRR: 0.51 [95\% CI: 0.31-0.84]). Recurrent perforation ( $>1$ episode) occurred in $8 \%$ (8/97) of vaccinees and $22 \%$ (11/51) of comparison participants (odds ratio: 0.33 [95\% CI: 0.11-1.00]) (Table 5).

A lower risk in vaccinees of new perforation, but not AOM, persisted throughout approximately 300 days of follow-up (Figure 3).

\section{Bilateral and Recurrent Tympanic Membrane Perforation} The number of participants experiencing unilateral or bilateral perforation and single or multiple episodes of perforation were analysed by log-linear modelling. Odds of both left and right TMP during follow-up in vaccinees were 0.41 [95\% CI: 0.18-0.94) compared to comparison participants. Similarly, vaccinees had lower odds of multiple perforations 0.40 [95\% CI: 0.18-0.87).

\section{Microbiology Outcomes}

Discharge from new perforations was collected more often from vaccinees than comparison participants (Table 6). Proportions of positive specimens (S. pneumoniae, $H$. 
Table 4: Univariate and multivariate survival analyses of time to first event for different otitis outcomes, hazard ratios and $95 \%$ confidence intervals

\begin{tabular}{|c|c|c|c|c|}
\hline \multirow[t]{2}{*}{ Variable/s included in analyses } & OME & AOM & TMP & CSOM \\
\hline & $\begin{array}{l}\text { Hazard ratio } \\
(95 \% \mathrm{Cl})\end{array}$ & $\begin{array}{l}\text { Hazard ratio } \\
(95 \% \mathrm{Cl})\end{array}$ & $\begin{array}{l}\text { Hazard ratio } \\
(95 \% \mathrm{Cl})\end{array}$ & $\begin{array}{l}\text { Hazard ratio } \\
(95 \% \mathrm{Cl})\end{array}$ \\
\hline \multicolumn{5}{|l|}{ Univariate } \\
\hline Vaccinated versus comparison group & $1.17(0.85-1.62)$ & $0.80(0.5 I-1.26)$ & $0.84(0.42-1.67)$ & $0.57(0.20-1.62)$ \\
\hline Age at first examination (days) & $0.98(0.98-0.99)$ & $1.00(0.99-1.01)$ & $\mathrm{n} / \mathrm{a}$ & $\mathrm{n} / \mathrm{a}$ \\
\hline Females/Males & $0.79(0.57-1.10)$ & $1.06(0.70-1.60)$ & $0.99(0.53-1.88)$ & $0.36(0.11-1.13)$ \\
\hline Rate of non-respiratory illness* & $1.09(0.79-1.52)$ & $1.08(0.73-1.60)$ & $1.07(0.57-2.01)$ & $0.98(0.39-2.46)$ \\
\hline Rate of prior antibiotic prescription* & $0.71(0.48-1.03)$ & $0.91(0.62-1.35)$ & $0.95(0.53-1.72)$ & $1.63(0.75-3.58)$ \\
\hline Birth weight (kg) & $0.99(0.74-1.33)$ & $\mathrm{I} .04(0.7 \mathrm{I}-\mathrm{I} .54)$ & $0.90(0.49-1.66)$ & $0.94(0.35-2.54)$ \\
\hline Rate of well clinic visits* & $1.10(0.99-1.23)$ & $1.32(1.07-1.62)$ & $1.09(0.74-1.62)$ & $1.10(0.57-2.12)$ \\
\hline Late immunisation & $0.64(0.36-1.17)$ & $0.69(0.39-1.22)$ & $0.66(0.31-1.40)$ & $1.19(0.40-3.54)$ \\
\hline \multicolumn{5}{|l|}{ Multivariate } \\
\hline Vaccinated versus comparison group & $0.90(0.64-1.25)$ & $1.01(0.61-1.67)$ & $0.83(0.41-1.66)$ & $0.56(0.19-1.62)$ \\
\hline Age at first examination (days) & $0.98(0.97-0.99)$ & $\mathrm{n} / \mathrm{a}$ & $\mathrm{n} / \mathrm{a}$ & $\mathrm{n} / \mathrm{a}$ \\
\hline Rate of non-respiratory illness* & $1.14(0.83-1.56)$ & $\mathrm{I} .04(0.7 \mathrm{I}-\mathrm{I} .52)$ & $1.09(0.58-2.07)$ & $1.04(0.42-2.60)$ \\
\hline Rate of well clinic visits* & $\mathrm{n} / \mathrm{a}$ & $1.32(1.05-1.65)$ & $\mathrm{n} / \mathrm{a}$ & $\mathrm{n} / \mathrm{a}$ \\
\hline
\end{tabular}

Note: 150 episodes of first OME in 160 participants, 91 episodes of first AOM in I27 participants, 38 episodes of first TMP in 135 participants, 15 episodes of first CSOM in 145 participants.

* (per month).

n/a not applicable.

influenzae or M. catarrhalis) were similar in the two groups. Proportions of positive specimens with serotypes included in the 7PCV and serotype $6 \mathrm{~B}$ were lower in vaccinees (Table 6). Predominant serotypes in the comparison group were $6 \mathrm{~B}$ ( 5 cases), 23F, 19A, 16F and $11 \mathrm{~A}(2$ each). In the vaccinated group $19 \mathrm{~A}, 19 \mathrm{~F}$ and $16 \mathrm{~F}$ were predominant (4 each), followed by 6B, 6A and 7C (2 each). In the vaccinated group, vaccine-type pneumococci were associated with 7 episodes of perforation which were distributed evenly throughout the duration of the study (data not shown).

\section{Discussion}

This is the first report of the impact of pneumococcal vaccination on any TMP and the natural history of OM in a high risk population. Introduction of vaccination was not associated with large effects on the outcomes of primary interest, delayed onset of OME and proportions of participants experiencing TMP. We also found that infant pneumococcal vaccination in remote Aboriginal communities was associated with, albeit with marginal significance:

a) Little effect on cumulative proportions of participants experiencing, or time to first episode of OME, AOM, TMP, or CSOM.

b) A reduced proportion developing CSOM at 9 months of age. c) Trends towards reduced incidence of AOM and TMP in the first 2 years of life.

d) Reduced recurrence of TMP.

In addition, the consistent relationship of cumulative hazards for TMP between the groups, suggested that any effect of three doses of 7PCV did not wane before 12 months of age.

The lack of substantial benefit of pneumococcal vaccination for OM in this population is likely due to a combination of factors. Vaccination had little effect on the high prevalence of $H$. influenzae infection or co-infections of $H$. influenzae and S. pneumoniae. As with studies in Finland and the US, vaccination did not prevent otitis associated with serotypes 19F[7,8] and 19A[7] which were common in our study. Unlike Finnish[7] and Czech Republic[20] studies of AOM, our data indicate a trend towards increased risk of TMP associated with vaccine-related serotypes. The US also reports increased proportions of AOM associated with vaccine-related and non-vaccine serotypes in the post-7PCV era $[21,22]$. Increased invasive 19A disease has been noted in post-7PCV surveillance among Alaska native[23] and Massachusetts children[24]. Although our findings do not support substantial replacement OM disease due to $H$. influenzae, they are consistent with US reports documenting potential $H$. influenzae 

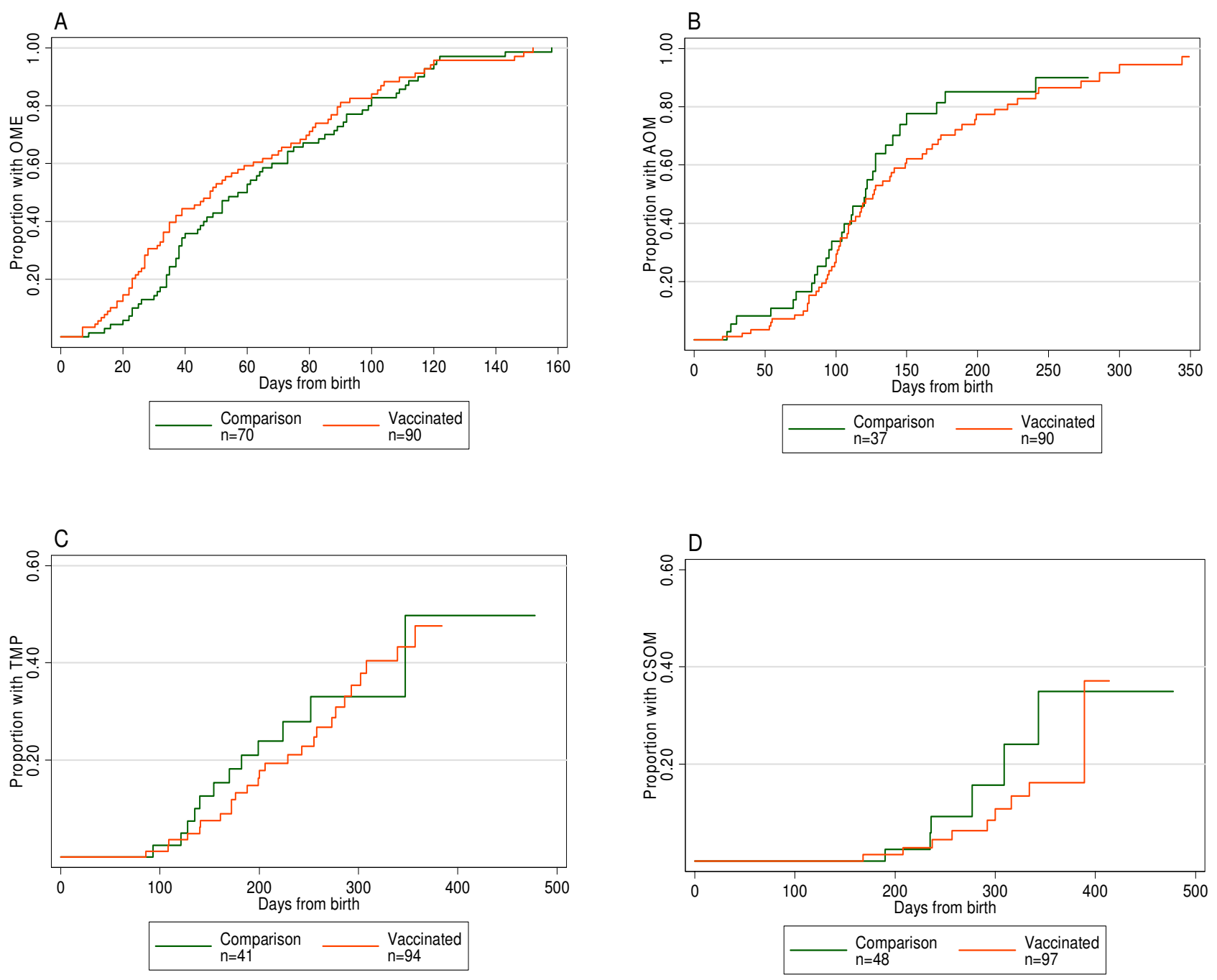

Figure 2

Kaplan-Meier curves for time to first event. Time to first: OME (A), AOM (B), TMP (C) and CSOM (D).

replacement disease following 7PCV $[22,25,26]$. Limited serotype coverage of $7 \mathrm{PCV}$ ( $50 \%$ of comparison group perforations) also contributed to poor vaccine effectiveness. Finally, early age of onset $(90 \%$ experienced OME and $50 \%$ experienced AOM by age 4 months; before the second dose of 7PCV) largely precludes an immunological response to vaccination affecting development of OM. Nonetheless, our finding that vaccinated children had fewer recurrent episodes of TMP suggests that a schedule stimulating an immune response before ear disease is established, e.g. maternal, neonatal or young infant dosing, may be more effective than a 2, 4, 6 month schedule. Although our study did not follow children for a sufficient duration to examine the effect of the 23PPV booster, immunogenicity has been demonstrated[27] with the potential to widen the serotype coverage beyond the 7 serotypes included in the 7PCV. Earlier administration of the booster may be more effective than the 18 month dose although it remains unknown whether boosting with the $7 \mathrm{PCV}$ or 23PPV is more effective.

Our study was designed to detect expected large effects. Our results, are however, consistent with more modest effects which would still be important in this population. A further limitation is the use of historic comparisons, which raises the possibility of bias or confounding. Universal vaccination precluded the use of concurrent controls. Although there are limitations, the study setting and measures we have taken, minimise the limitations. Bias due to temporal changes is limited as historic data (19962001) are continuous with data collected after vaccine introduction (2001-2004). Ecological data suggested reduced household crowding over time but no change in levels of income, unemployment or the proportion of 
Table 5: Incidence of AOM and TMP in vaccinated and comparison groups, including sensitivity analyses

\begin{tabular}{|c|c|c|c|c|c|c|c|c|}
\hline & \multicolumn{4}{|c|}{ AOM } & \multicolumn{4}{|c|}{ TMP } \\
\hline & \multicolumn{2}{|c|}{ Baseline analysis } & \multicolumn{2}{|c|}{ Sensitivity analysis } & \multicolumn{2}{|c|}{ Baseline analysis } & \multicolumn{2}{|c|}{ Sensitivity analysis } \\
\hline & Vaccinated & Comparison & Vaccinated & Comparison & Vaccinated & Comparison & Vaccinated & Comparison \\
\hline No. of participants & 90 & 37 & 97 & 51 & 94 & 41 & 97 & 51 \\
\hline $\begin{array}{l}\text { Examinations per } \\
\text { year }\end{array}$ & 11.53 & 11.80 & 10.24 & 10.20 & 10.77 & 11.70 & 10.03 & 10.20 \\
\hline No. of episodes & 104 & 45 & 139 & 87 & 35 & 23 & 37 & 33 \\
\hline $\begin{array}{l}\text { No. with } 0 \\
\text { episodes (\%) }\end{array}$ & $29(32)$ & $9(24)$ & $24(25)$ & $6(12)$ & $70(74)$ & $30(73)$ & 71 (73) & $34(67)$ \\
\hline $\begin{array}{l}\text { No. with I episode } \\
\text { (\%) }\end{array}$ & $28(3 I)$ & $14(38)$ & $28(29)$ & $17(33)$ & $16(17)$ & $3(7)$ & $18(19)$ & $6(12)$ \\
\hline $\begin{array}{l}\text { No. with >1 } \\
\text { episodes (\%) }\end{array}$ & $33(37)$ & $14(38)$ & $44(45)$ & $28(55)$ & $8(8)$ & $8(19)$ & $8(8)$ & II (22) \\
\hline $\begin{array}{l}\text { Unadjusted } \\
\text { incidence }\end{array}$ & $1.87 / y r$ & $2.05 / \mathrm{yr}$ & $1.68 / \mathrm{yr}$ & $2.02 / y r$ & $0.55 / \mathrm{yr}$ & $0.82 / y r$ & $0.44 / y r$ & $0.77 / y r$ \\
\hline Adjusted incidence* & $1.83 / \mathrm{yr}$ & $2.03 / y r$ & $1.62 / y r$ & $2.02 / y r$ & $0.47 / y r$ & $0.75 / y r$ & $0.39 / \mathrm{yr}$ & $0.75 / y r$ \\
\hline $\begin{array}{l}\text { Absolute rate } \\
\text { reductiont }\end{array}$ & $-0.20 / y r$ & & $-.40 / y r$ & & $-0.28 / y r$ & & $-0.36 / y r$ & \\
\hline
\end{tabular}

* Adjusted in multivariate poisson model considering repeated events.

† Absolute rate reduction is the incidence in the vaccinated minus incidence in the comparison group.

Baseline analyses of incidence of AOM and TMP included participants if the first examination was at less than 4 months and less than 6 months respectively, and included follow-up data if repeated examinations were less than 2 months apart. Sensitivity analyses included participants with any age at first examination, and included follow-up data if repeated examinations were less than 4 months apart.

young children in the population. Bias towards a positive vaccine effect due to temporal improvements in child care practices, health services (e.g. more intensive antibiotic schedules) and environmental conditions is possible, however this was not evident from clinical characteristics before 6 months of age (Tables 1 \&2), nor from the essentially non-significant effect that was observed. Potential confounding is reduced as otitis risk factors are universal in this population: breast feeding, absence of pacifiers, family history of otitis and extremely high rates of smok- ing. Study procedures were objective and held constant throughout the study period. We partially adjusted for potential bias of historic comparisons by adjusting for rates of non-respiratory illness as a surrogate measure of general ill health. Enrolment of $80 \%$ of the population in the vaccinated group and 54\% in the comparison group, who were also involved in OM-RCT with more restrictive inclusion criteria and supervised therapy, introduced selection bias against a positive vaccine effect.

Table 6: Pathogens isolated from new tympanic membrane perforations

\begin{tabular}{|c|c|c|}
\hline Perforation pathogen category & $\begin{array}{c}\text { Vaccinated } \\
N=97(194 \text { ears })\end{array}$ & $\begin{array}{c}\text { Comparison } \\
N=51 \text { (102 ears) }\end{array}$ \\
\hline New perforations & 73 (38\% of ears) & 72 (7I\% of ears) \\
\hline New perforation discharge specimen collected & $59 / 73$ (81\% new perforations) & $44 / 72$ (61\% new perforations) \\
\hline Positive discharge specimens* & $32 / 59$ (54\% specimens) & $25 / 44$ (57\% specimens) \\
\hline S. pneumoniae & 19/32 (59\% positive specimens) & 16/25 (64\% positive specimens) \\
\hline All vaccine-types & $7(22 \%)$ & $8(32 \%)$ \\
\hline Serotype 6B & $2(3 \%)$ & $5(20 \%)$ \\
\hline All vaccine-related types & $6(19 \%)$ & $2(8 \%)$ \\
\hline Serotype 19A & $4(13 \%)$ & $2(8 \%)$ \\
\hline All non-vaccine types & $9(28 \%)$ & $6(24 \%)$ \\
\hline Serotype I6F & $4(13 \%)$ & $2(8 \%)$ \\
\hline H. influenzae & $28(88 \%)$ & $21(84 \%)$ \\
\hline M. catarrhalis & $0(0 \%)$ & $2(8 \%)$ \\
\hline S. pneumoniae \& $H$. influenzae ${ }^{\dagger}$ & $15(47 \%)$ & $12(48 \%)$ \\
\hline
\end{tabular}

* Isolation of $S$. pneumoniae, $H$. influenzae or $M$. catarrhalis.

t Cases of co-infections of $H$. influenzae and $S$. pneumoniae have also been included in the numbers for individual pathogens.

Other serotypes isolated: vaccinated group I9F (3), 6A (2), 7C (2), 23F, 3, 7F, I \& I9F co-isolated. comparison group $23 \mathrm{~F}(2)$, IIA (2), 9V, 7F, I3. 

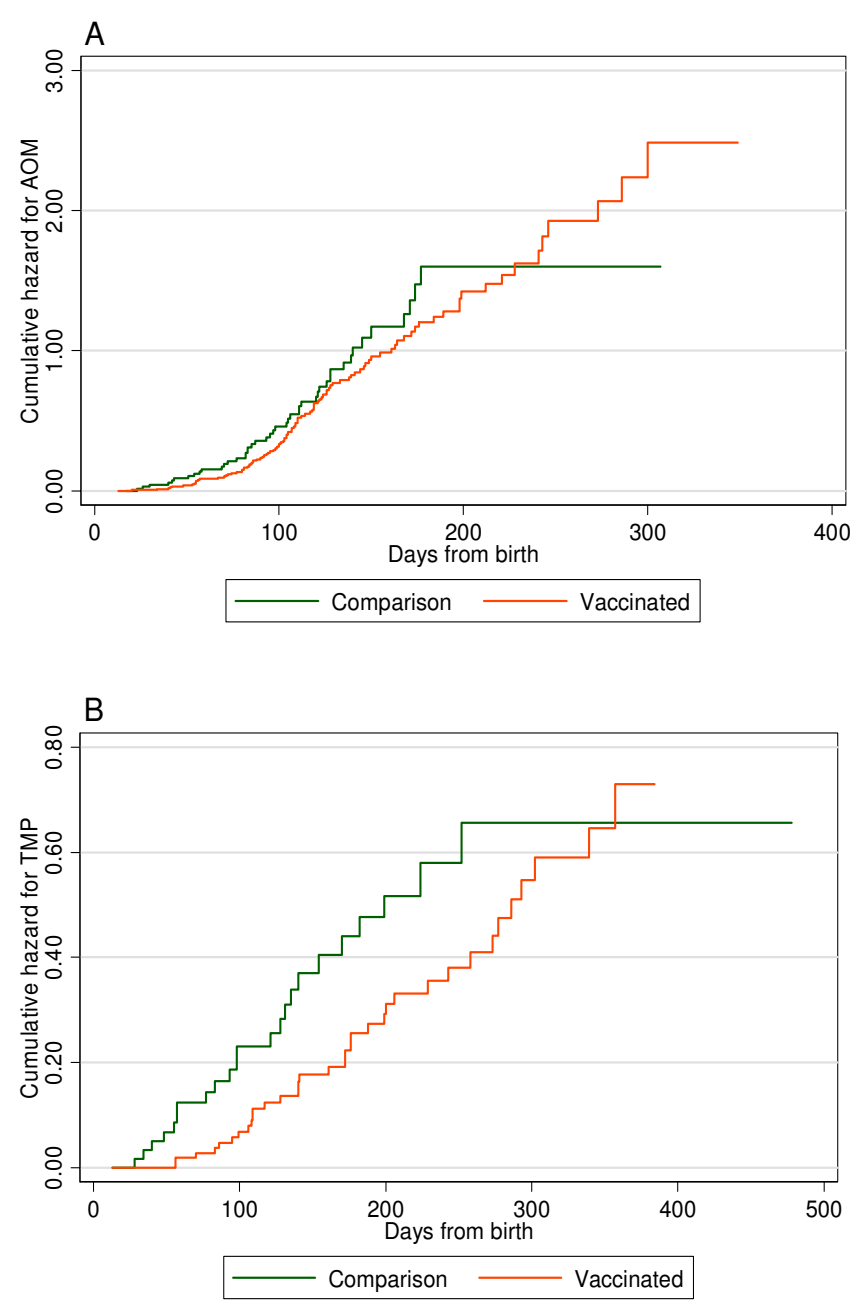

Figure 3

Cumulative hazard curves for events of $A O M(A)$ and new TMP (B).

\section{Conclusion}

Due to potential confounding and bias, the results of this study should be interpreted with caution. Despite the association of infant pneumococcal vaccination with possible reductions in incidence of AOM and TMP, the cumulative proportion of Aboriginal children experiencing OME, AOM, TMP and CSOM was unchanged as was the time to development of different OM outcomes. Of note, the lack of vaccine effectiveness to substantially reduce proportions of participants experiencing different OM outcomes and delayed onset of OM does not imply evidence of no effect. This vaccination is unlikely to reduce the prevalence of ear disease in this population in the first years of life. Our findings have important implications for the generalisability of research conducted in affluent populations for high-risk disadvantaged populations. This assertion is supported by data from Navajo and White Mountain Apache infants, where 7PCV efficacy against clinically-diagnosed and severe OM was somewhat lower[10] than in Californian[6] and Finnish[7] children. Results of post-7PCV licensure studies among affluent populations regarding OM[26,28,29], pneumonia[30] and invasive disease[31] have been very positive. However, 7PCV may have little effect against simple and suppurative ear disease and pneumonia[32] in populations at very high risk of pneumococcal infection and those living in disadvantaged conditions which attribute such risk. Further study of infant cohorts is necessary to document longer term vaccine effectiveness which incorporates potentially increasing indirect effects over time. Thus, the results of efficacy studies and licensing and use of a 10valent pneumococcal-Haemophilus protein D conjugate vaccine, similar to a product shown effective against OM in Europe[20], and a 13-valent PCV[33] are much anticipated. Post-introduction studies of sufficient size, will be needed to better define the impact of these vaccines against complicated $\mathrm{OM}$ in the Aboriginal population. Apart from wider serotype coverage (particularly serotypes 19A, 6A and 16F), other potential avenues to improve the effectiveness of pneumococcal vaccines in this population include: maternal, neonatal or young infant vaccination and development of common pneumococcal antigen vaccines. Vaccines with efficacy against OM due to multiple pathogens are also needed. In the meantime, there should be ongoing focus on early detection and treatment of ear disease and hearing impairment, and the underlying, predominantly social and economic, determinants of ear infections in Aboriginal Australians.

\section{Competing interests}

The authors declare that they have no competing interests.

\section{Authors' contributions}

GM joined the study in February 2002, and participated in field work, data collection and entry as well as developing the analysis plan, performing data analysis and writing the manuscript with input from JC, PM and AL. JC assisted in analysis and provided input on the writing of the manuscript. AL developed the study protocol, wrote the funding grant applications, supervised field and laboratory work, assisted in analysis and provided input on the writing of the manuscript. PM developed the study protocol, wrote the funding grant applications, supervised field work, assisted in analysis and provided input on the writing of the manuscript. All authors read and approved the final manuscript.

\section{Acknowledgements}

Priscilla Tipakalippa, Chris Wigger and Grant Mackenzie were the primary field workers with assistance from Una Pilakui, Edna Gadil, J R Gadil, Gabrielle Mellon and Kate Wilson. Elizabeth Stubbs, Kim Hare, Brooke Hanson and Amanda Kennedy performed the laboratory work. Jemima Beissbarth was involved in laboratory work and assisted Robyn Liddle with data man- 
agement. Al Yonovitz gave technical assistance with the video otoscopy equipment.

We acknowledge the children, families and Tiwi communities who were involved in the studies. We thank the community leaders, the Tiwi health board and the staff of local health clinics for their support. The study was funded by the National Health and Medical Research Council of Australia and Wyeth Australia. Menzies School of Health Research staff and facilities were essential to the success of the study. We thank the Cooperative Research Centre for Aboriginal and Tropical Health for valuable support.

Dr Mackenzie received a scholarship from the National Health \& Medical Research Council of Australia.

This work is attributed to the Child Health Division, Menzies School of Health Research.

\section{References}

I. Morris PS, Leach AJ, Silberberg P, Mellon G, Wilson C, Hamilton E, Beissbarth J: Otitis media in young Aboriginal children from remote communities in Northern and Central Australia: a cross sectional survey. BMC Pediatrics 2005, 5:27.

2. Rothstein J, Heazlewood R, Fraser M: Health of Aboriginal and Torres Strait Islander children in remote Far North Queensland: findings of the Paediatric Outreach Service. MJA 2007, | 86:519-52|.

3. WHO/CIBA Foundation: Prevention of hearing impairment from chronic otitis media. Presented at a WHO/CIBA Foundation Workshop. 1996:19-2I [http://www.noiseandhealth.orarticle.asp? issn $=|463-174|$;year $=1998$; vol ume $=I ;$ issue $=1 ;$ spage $=6 ;$ epage $=12$; aulast $=$ Smith $]$.

4. Leach AJ, Mackenzie GA, Hare K, Stubbs E, Beissbarth J, Kennedy M, Wigger C, Gadil JR, Morris P: Microbiology of acute otitis media with perforation (AOMwiP) in Aboriginal children living in remote communities - monitoring the impact of 7-valent pneumococcal conjugate vaccine (7vPCV). In Streptococci: New Insights into an Old Enemy: 25-29 September 2005; Palm Cove Edited by: Sriprakash KS. Elsevier Science: Philadelphia; 2006:89-92.

5. Leach AJ: Prospective studies of respiratory pathogens, particularly Streptococcus pneumoniae, in Aboriginal and nonAboriginal infants: impact of antibiotic use and implications for otitis media. In PhD thesis University of Sydney; 1996.

6. Fireman B, Black SB, Shinefield HR, Lee J, Lewis E, Ray P: Impact of the pneumococcal conjugate vaccine on otitis media. Pediatr Infect Dis J 2003, 22:10-16.

7. Eskola J, Kilpi T, Palmu A, Jokinen J, Haapakoski J. Herva E, Takala A, Kayhty H, Karma P, Kohberger R, Siber G, Makela PH: Efficacy of a pneumococcal conjugate vaccine against acute otitis media. N Engl J Med 200I, 344:403-409.

8. Black S, Shinefield H, Fireman B, Lewis E, Ray P, Hansen JR, Elvin L, Ensor KM, Hackell J, Siber G, Malinoski F, Madore D, Chang I, Kohberger R, Watson W, Austrian R, Edwards K: Efficacy, safety and immunogenicity of heptavalent pneumococcal conjugate vaccine in children. Pediatr Infect Dis / 2000, I 9:187-195.

9. Palmu AA, Verho J, Jokinen J, Karma P, Kilpi TM: The seven-valent pneumococcal conjugate vaccine reduces tympanostomy tube placement in children. Pediatr Infect Dis / 2004, 23:732-738.

10. O'Brien KL, David AB, Chandran A, Moulton LH, Reid R, Weatherholtz R, Santosham M: Randomized, controlled trial efficacy of pneumococcal conjugate vaccine against otitis media among Navajo and White Mountain Apache infants. Pediatr Infect Dis 2008, 27:7|-73

II. Minja BM, Moshi NH, Ingvarsson L, Bastos I, Grenner J: Chronic suppurative otitis media in Tanzanian school children and its effects on hearing. East Afr Med / 2006, 83:322-325.

12. Kamal N, Joarder AH, Chowdhury AA, Khan AW: Prevalence of chronic suppurative otitis media among the children living in two selected slums in Dhaka City. Bangladesh Med Res Counc Bull 2004, 30:95-104.

13. Amusa YB, ljadunola IK, Onayade OO: Epidemiology of otitis media in a local tropical African population. West Afr J Med 2005, 24:227-230.
14. Matanda RN, Muyunga KC, Sabue MJ, Creten W, Heyning P Van de: Chronic suppurative otitis media and related complications at the University Clinic of Kinshasa. B-ENT 2005, 1:57-62.

15. Australian Bureau of Statistics: ABS Website. 200I Census doc. no. 71010 Bathurst-Melville (Statistical Local Area) 2002 [http://www.cen susdata.abs.gov.au/ABSNavigation/prenav/PopularAreas\&collec tion=Census\&period $=200$ I \&\&navmapdisplayed=true\&textversion=false]. ABS; Canberra

16. Leach AJ, Morris PS, Mathews JD: Compared to placebo, longterm antibiotics resolve otitis media with effusion and prevent acute otitis media with perforation in a high-risk population: a randomized double-blind placebo controlled trial. BMC Pediatrics 2008, 8:23 [http://www.biomedcentral.com/|47|2431/8/23].

17. O'Brien KL, Nohynek H, The WHO Pneumococcal Vaccine Trials Carriage Working Group: Report from a WHO Working Group: standard method for detecting upper respiratory carriage of Streptococcus pneumoniae. Pediatr Infect Dis J 2003, 22: $133-140$

18. Leach AJ, Boswell JB, Asche V, Nienhuys TG, Mathews JD: Bacterial colonization of the nasopharynx predicts very early onset and persistence of otitis media in Australian Aboriginal infants. Pediatr Infect Dis J 1994, I 3:983-989.

19. Australian Bureau of Statistics: ABS Website. 1996 Census doc. no. 7/0100609 Bathurst-Melville (Statistical Local Area) 1997 [http:www.abs.gov.au/AUSSTATS/abs@.nsf/96cdbygeogtype?open view\&restricttocategory=Main 20Areas\&Expand=1\&]. ABS; Canberra

20. Prymula R, Peeters P, Chrobok V, Kriz P, Novakova E, Kaliskova E, Kohl I, Lommel P, Poolman J, Prieels JP, Schuerman L: Pneumococcal capsular polysaccharides conjugated to protein $D$ for prevention of acute otitis media caused by both Streptococcus pneumoniae and non-typable Haemophilus influenzae: a randomised double-blind efficacy study. Lancet 2006, 367:740-748.

21. McEllistrem MC, Adams JM, Patel K, Mendelsohn AB, Kaplan SL, Bradley JS, Schutze GE, Kim KS, Mason EO, Wald ER: Acute otitis media due to penicillin-nonsusceptible Streptococcus pneumoniae before and after the introduction of the pneumococcal conjugate vaccine. Clin Infect Dis 2005, 40: I738-I744.

22. Pichichero ME, Casey JR: Emergence of a multiresistant serotype I9A pneumococcal strain not included in the 7-valent conjugate vaccine as an otopathogen in children. JAMA 2007, 298: I772- 1778

23. Singleton RJ, Hennessy TW, Bulkow L, Hammitt LL, Zulz T, Hurlburt DA, Butler JC, Rudolph K, Parkinson A: Invasive pneumococcal disease caused by nonvaccine serotypes among Alaska Native children with high levels of 7-valent pneumococcal conjugate vaccine coverage. JAMA 2007, 297: I784-I792.

24. Pelton SI, Huot H, Finkelstein JA, Bishop C], Hsu KK, Kellenburg J, Huang SS, Goldstein R, Hanage WP: Emergence of I9A as a virulent and multidrug resistant pneumococcus in Massachusetts following universal immunization of infants with pneumococcal conjugate vaccine. Pediatr Infect Dis J 2007, 26:468-472

25. Block SL, Hedrick J, Harrison C], Tyler R, Smith A, Findlay R, Keegan $\mathrm{E}$ : Community-wide vaccination with heptavalent pneumococcal conjugate significantly alters the microbiology of acute otitis media. Pediatr Infect Dis J 2004, 23:829-833.

26. Casey JR, Pichichero ME: Changes in frequency and pathogens causing acute otitis media in 1995-2003. Pediatr Infect Dis J 2004, 23:824-828.

27. Leach AJ, Morris PS, Mackenzie G, McDonnell J, Balloch A, Carapetis J, Tang M: Immunogenicity for 16 serotypes of a unique schedule of pneumococal vaccines in a high-risk population. Vaccine 2008, 26:3885-3890.

28. Poehling KA, Szilagyi PG, Grijalva CG, Martin SW, Lafleur B, Mitchel $E$, Barth RD, Nuorti JP, Griffin MR: Reduction of frequent otitis media and pressure-equalizing tube insertions in children after introduction of pneumococcal conjugate vaccine. Pediatrics 2007, I | 9:707-7I5.

29. Fletcher MA, Fritzell B: Brief review of the clinical effectiveness of PREVENAR(R) against otitis media. Vaccine 2006, 25:2507-25I 2.

30. Grijalva CG, Nuorti JP, Arbogast PG, Martin SW, Edwards KM, Griffin $M R$ : Decline in pneumonia admissions after routine child- 
hood immunisation with pneumococcal conjugate vaccine in the USA: a time-series analysis. Lancet 2007, 369: I I79-I I 86.

31. Whitney CG, Farley MM, Hadler J, Harrison LH, Bennett NM, Lynfield R, Reingold A, Cieslak PR, Pilishvili T, Jackson D, Facklam RR, Jorgensen $\mathrm{JH}$, Schuchat $\mathrm{A}$ : Decline in invasive pneumococcal disease after the introduction of protein-polysaccharide conjugate vaccine. $N$ Engl J Med 2003, 348: I737-1746.

32. O'Grady KA, Taylor-Thomson D, Chang A, Torzillo P, Morris P, Mackenzie G, Wheaton G, Bauert P, De Campo M, De Campo J, Ruben A: The burden of hospitalised, radiologically diagnosed pneumonia in Northern Terriotory Indigenous children in Australia. ISPPD-6 6th International Symposium on Pneumococci and Pneumococcal Diseases: 8-12 June 2008; Reykjavik 2008:SOI-03.

33. Wyeth product pipeline: Wyeth website [http:// www.wyeth.com/research/projects]

\section{Pre-publication history}

The pre-publication history for this paper can be accessed here:

http://www.biomedcentral.com/1471-2431/9/14/prepub

Publish with Bio Med Central and every scientist can read your work free of charge

"BioMed Central will be the most significant development for disseminating the results of biomedical research in our lifetime."

Sir Paul Nurse, Cancer Research UK

Your research papers will be:

- available free of charge to the entire biomedical community

- peer reviewed and published immediately upon acceptance

- cited in PubMed and archived on PubMed Central

- yours - you keep the copyright

Submit your manuscript here:

http://www.biomedcentral.com/info/publishing_adv.asp 